\title{
Ternary clusterization and quadrupole deformation
}

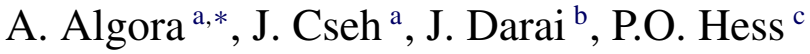 \\ ${ }^{a}$ Institute of Nuclear Research of the Hungarian Academy of Sciences, Pf. 51, Debrecen 4001, Hungary \\ b Institute of Experimental Physics, University of Debrecen, Bem tér 18/A, Debrecen 4026, Hungary \\ ${ }^{\mathrm{c}}$ Instituto de Ciencias Nucleares, UNAM, Circuito Exterior, C.U., A.P. 70-543, 04510 México, D.F., Mexico
}

Received 15 August 2005; received in revised form 8 February 2006; accepted 30 June 2006

Available online 18 July 2006

Editor: J.-P. Blaizot

\begin{abstract}
The deformation-dependence of ternary clusterization of atomic nuclei is investigated. In particular, allowed and forbidden ternary clusterconfigurations are determined for the ground, superdeformed, and hyperdeformed states of some light and heavy nuclei, based on a microscopic (real and effective $S U(3)$ ) selection rule. The energetic stability of the clusterizations is also considered.

(C) 2006 Elsevier B.V. All rights reserved.
\end{abstract}

PACS: 21.60.Fw; 21.60.Gx

Keywords: Ternary clusterization; Pauli-principle; Energetic preference

The investigation of exotic nuclear shapes, e.g. superdeformed and hyperdeformed states is one of the most interesting topics in recent nuclear structure studies. The appearance of exotic cluster configurations (or exotic cluster decay) is another issue of utmost interest. The combination of these two problems brings us to an exciting question: what is the interrelation of these two phenomena, i.e. what are the possible clusterizations of nuclear states with exotic shapes. In the present Letter we address this question from the angle of the ternary clusterization. We apply methods which can be generalized to more complicated multicluster-configurations in a straightforward way.

The basic concept of this work is that when we try to describe the composition of an atomic nucleus from smaller nuclei (clusters) then we take into account both of the two complementary natural laws, which govern this kind of phenomenon: the energy-minimum principle and the Pauli-exclusion principle. The crucial role of these two rules are obvious: energetically unfavored systems are not likely to appear, and when the building blocks are fermions, like the nucleons of the atomic nuclei, then they follow the exclusion principle. However, the exact role, or

\footnotetext{
* Corresponding author.

E-mail address: algora@atomki.hu (A. Algora).
}

relative importance of these two aspects of clusterization among different circumstances are not completely understood yet; the present work is meant to be a contribution to this task.

Much attention has been paid to the energetic preference of various cluster-configurations of a nucleus. The methods applied along this line are partly or completely empirical ones, using information gained from the experimental data. Furthermore, most of these works concentrate on the simplest, i.e. binary clusterizations, especially, when the energetic calculation involves (in addition to the experimental binding energies) intercluster potentials, like in the dinuclear system model [1], or in the local potential approach [2]. On the other hand the treatment of the exclusion-principle has to be done microscopically, therefore, apart from the light, or simplest heavy nuclei, it gives rise to large computational difficulties. Due to this fact no systematic studies has been done, and many of the experimentally interesting systems are untouched from this viewpoint.

The main point of this Letter is to present a method for the approximative treatment of the exclusion principle, which can be applied both to binary [3] and to ternary (and even to multi) cluster-configurations. The results of these calculations can be combined with those on the energetic stability. As for this latter quantity, it can be determined within different approximations. One of the simplest possibility is provided 
by the binding-energy argument, as it is discussed by Buck et al. [4]. This procedure does not contain, however, any details of the cluster-cluster interactions, which are obviously important. On the other hand, they are treated carefully (both for the Coulombic and for the effective nucleon-nucleon interactions) in e.g. the dinuclear system model [1]. In order to reach the final conclusions on the possible cluster-configurations calculations including these details need to be done, of course; and their conclusions have to be combined with those on the exclusion principle. Up to our knowledge, energetic calculations of this kind are not available at present for the ternary clusterizations discussed here. Therefore, we combine the results of our selection-rule calculations with those of the bindingenergy, in light of its simplicity. (We obtain it in a way similar to the work of Buck et al. [4], except for a straightforward generalization from binary to ternary clusterization.) In doing so our intention is not to pretend that the final conclusion on the cluster-preference is obtained, rather we wish to emphasize the need for the combination of the calculations of energetic stability and exclusion principle.

In this way both aspects of the clusterization can be handled, therefore, their interrelation can be studied in specific problems. The exclusion-principle is taken into account by a selection rule, based on the real [5] or effective [6] $U(3)$ symmetry for light and heavy nuclei, respectively. This symmetry-based consideration can also be very involved for heavy nuclei, nevertheless, it seems to be widely applicable.

In comparison with other cluster studies the novel feature of our work is that we address problems which has been investigated so far (in a systematic manner) only with phenomenologic approaches $[1,2]$. We present here a method which enables us to incorporate the most important aspect of the microscopic description, i.e. the Pauli-exclusion principle, in an approximate but systematically applicable way. Our description is not fully microscopic rather it can be called semimicroscopic, but it reaches a territory which is hopeless for the fully microscopic calculations at present. It turns out that the incorporation of the exclusion principle together with the appropriate treatment of the deformation and orientation of the clusters within the nuclear molecular states brings us to physical conclusions, which are new and unexpected based on our previous experiences from the phenomenological models.

As specific examples we consider ternary cluster-configurations in the ground, superdeformed and hyperdeformed states of the light ${ }^{36} \mathrm{Ar}$ and heavy ${ }^{252} \mathrm{Cf}$ nuclei, which were investigated from the viewpoint of possible binary clusterization in [3]. In case of ${ }^{36} \mathrm{Ar}$ the superdeformed state has been found experimentally [7], and a theoretical prediction is available for its hyperdeformed state [8]. In case of ${ }^{252} \mathrm{Cf}$ the main motivation is provided by the spontaneous fission experiments from its ground state, which indicated several very exotic clusterizations [9]. As for superdeformed and hyperdeformed states of this nucleus, we consider the results of the Nilsson-model with appropriate quadrupole deformation [3].

The structural selection rule we apply, is based on the $U(3)$ symmetry, what is known to be a good approximate symmetry of light nuclei [5], and its role in the clusterization was also ob- served in the early studies [10], followed by the understanding of its importance from different aspects of clusterization [11]. For a ternary cluster-configuration there are two independent relative motions (along the corresponding Jacobi-coordinates), therefore, the $U(3)$ selection rule reads:

$$
\begin{aligned}
& {\left[n_{1}, n_{2}, n_{3}\right]} \\
& \quad=\left[n_{1}^{(1)}, n_{2}^{(1)}, n_{3}^{(1)}\right] \otimes\left[n_{1}^{(2)}, n_{2}^{(2)}, n_{3}^{(2)}\right] \\
& \quad \otimes\left[n_{1}^{(3)}, n_{2}^{(3)}, n_{3}^{(3)}\right] \otimes\left[n^{\left(R_{1}\right)}, 0,0\right] \otimes\left[n^{\left(R_{2}\right)}, 0,0\right],
\end{aligned}
$$

where $\left[n_{1}, n_{2}, n_{3}\right]$ is the set of (approximate) $U(3)$ quantum numbers of the parent nucleus, the superscript $(i)$ stands for the $i$ th cluster, and $\left(R_{i}\right)$ indicates relative motion (along the corresponding Jacobi-coordinates). This is a straightforward generalization of the $U(3)$ selection rule applied to binary clusterizations (see e.g. [3,12], and references therein). Please, note that this coupling of the $U(3)$ quantum numbers is quite general, no assumption is made on a stretched, or maximally deformed configuration. In geometrical terms: the $U(3)$ representations characterize the deformation of the clusters (including triaxial shapes as well), and the general coupling without simplifying assumptions allows all the possible relative orientations of the clusters with respect to the molecular axis.

In medium and heavy nuclei, however, the $U(3)$ symmetry is not valid in its original form, due to the importance of the symmetry-breaking interactions, like spin-orbit and pairing. Nevertheless, it was found in [6] that in spite of the strong symmetry-breaking interactions a generalized $U(3)$ symmetry, called effective, or quasi-dynamical $U(3)$ symmetry, may survive even for heavy nuclei. It is very general in the sense that the energy-eigenfunctions are composed as linear combinations of basis states belonging to different $U(3)$ irreducible representations (irreps). Nevertheless, the mixing is a special one, it is a coherent mixing of different irreducible representations, which results in an approximate $U(3)$ symmetry. This symmetry is determined by effective, or average $U(3)$ quantum numbers. The physical condition for the special mathematical structure of the effective symmetry is the adiabatic decoupling of the single nucleon motion and the collective motion of the nucleus. In [13] a method was developed for the determination of the effective $U$ (3) quantum numbers of the heavy nuclei, based on the occupation of the asymptotic Nilsson orbits. The procedure, which was originally invented for the large prolate deformation was extended in [14] to the oblate shape and small deformations as well, based on the expansion of single-particle orbitals in terms of asymptotic Nilsson-states. Thus, in practical terms the determination of the effective $U(3)$ quantum numbers is done in the following way. (i) Starting from the quadrupole deformation of the cluster or parent nucleus we fill in the Nilsson-orbitals at the proper deformation. (ii) Then the single-particle states at this finite deformation is expanded into asymptotic Nilsson-states. (iii) The contribution of the asymptotic Nilsson-orbitals to the effective $U(3)$ quantum numbers are determined as discussed in $[13,14]$.

When applying the effective $U(3)$ symmetry in cluster studies, it describes an average (or effective) clusterization. It is worth stressing that the effective $U(3)$ symmetry, and the ef- 
fective $U(3)$ quantum numbers are valid to the same extent, like the concept of the quadrupole deformation of the nucleus in question. Both of them are determined by the coherent superposition of several basis states belonging to real $U(3)$ irreps of the many-fermion system [15]. The concept of effective symmetry is applicable also to light nuclei, and when the simple leading representation approximation is valid, the real and effective $U(3)$ quantum numbers usually coincide [14]. This circumstance gives a straightforward way for the extension of the simple selection rule consideration.

When a cluster configuration is forbidden, we can characterize its forbiddenness quantitatively $[3,16]$. In particular, a quantity $S$, called reciprocal forbiddenness, is calculated such that $0 \leqslant S \leqslant 1$. It measures the similarity between the $U(3)$ symmetry (or in geometrical terms: the shape) of the parent nuclear state and that of the cluster configuration, such that $S=0$, and $S=1$ correspond to completely forbidden and allowed clusterizations, respectively.

Concerning the relation of the reciprocal forbiddenness $(S)$, calculated here, to the microscopic cluster spectroscopic factor $(S F)$, the following can be said. They are not identical, of course, the cluster spectroscopic factor can be determined only from a fully microscopic calculation. Nevertheless, they have some similarities. Unlike the phenomenological models, our consideration based on $S$ reflects the non-orthogonal nature of different cluster-configurations, just like the microscopic spectroscopic factor. Furthermore, when $S=0$, i.e. the cluster configuration is forbidden according to our selection-rule consideration, then $S F=0$ for the $S U(3)$ state in question (exactly for light nuclei, and in a sort of average for heavy nuclei). If, however, the cluster-configuration is allowed $(S=1)$, then $S F$ still can be smaller than 1 . Please, note, that the microscopic definition of the spectroscopic factor (see e.g. [17]) is such, that even for a pure cluster state it is not necessarily 1 . Thus, in short we can say that our reciprocal forbiddenness $(S)$ is not identical, of course, with the microscopic spectroscopic factor, but it is related to it; in some sense it can be considered as its semimicroscopic approximation.

The criterium of maximal stability [4], which represents a complementary viewpoint for the selection of clusterization, requires the largest value of the summed differences of the measured binding energies and the corresponding liquid drop values. For the case of a ternary clusterization it reads:

$$
\begin{aligned}
D(1,2,3)= & {\left[B(1)-B_{L}(1)\right]+\left[B(2)-B_{L}(2)\right] } \\
& +\left[B(3)-B_{L}(3)\right],
\end{aligned}
$$

where $B(i)$ is the experimental binding energy of the $i$ th cluster [18], while $B_{L}(i)$ stands for liquid drop value [4].

In the generalized version of the method, as we apply it here, a further condition is also taken into account, which is called dipole constraint [4]. It is based on the observation that electric dipole transitions are weak, therefore, the decomposition is expected to be close to satisfying the constraint:

$\frac{Z_{T}}{A_{T}} \approx \frac{Z_{1}}{A_{1}} \approx \frac{Z_{2}}{A_{2}} \approx \frac{Z_{3}}{A_{3}}$,

where $T$ stands for total.
Table 1

Ternary clusterizations of ${ }^{36} \mathrm{Ar}$ including ${ }^{4} \mathrm{He}$ (upper part) and ${ }^{16} \mathrm{O}$ (lower part). $S$ stands for the reciprocal forbiddenness of the indicated states (ND: normal deformed, SD: superdeformed, HD: hyperdeformed). The $S$ values are identical for different cluster-configurations belonging to the same mass-fragmentation.

\begin{tabular}{|c|c|c|c|c|c|}
\hline \multirow[t]{2}{*}{$A_{1}+A_{2}$} & \multirow[t]{2}{*}{ Nuclei } & \multicolumn{3}{|c|}{$S$} & \multirow[t]{2}{*}{$D(1,2,3)$} \\
\hline & & ND & SD & HD & \\
\hline $16+16$ & ${ }_{8}^{16} \mathrm{O}+{ }_{8}^{16} \mathrm{O}$ & 0.070 & 0.169 & 1.000 & 17.59 \\
\hline \multirow{2}{*}{$18+14$} & \multirow{2}{*}{$\begin{array}{l}{ }_{10}^{18} \mathrm{Ne}+{ }_{6}^{14} \mathrm{C} \\
{ }_{8}^{18} \mathrm{O}+{ }_{8}^{14} \mathrm{O}\end{array}$} & \multirow[t]{2}{*}{0.131} & \multirow[t]{2}{*}{1.000} & \multirow[t]{2}{*}{1.000} & 12.88 \\
\hline & & & & & 12.45 \\
\hline $20+12$ & ${ }_{10}^{20} \mathrm{Ne}+{ }_{6}^{12} \mathrm{C}$ & 1.000 & 1.000 & 1.000 & 14.89 \\
\hline \multirow{2}{*}{$22+10$} & ${ }_{12}^{22} \mathrm{Mg}+{ }_{4}^{10} \mathrm{Be}$ & \multirow[t]{2}{*}{1.000} & \multirow[t]{2}{*}{1.000} & \multirow[t]{2}{*}{1.000} & 11.67 \\
\hline & ${ }_{10}^{22} \mathrm{Ne}+{ }_{6}^{10} \mathrm{C}$ & & & & 11.56 \\
\hline $24+8$ & ${ }_{12}^{24} \mathrm{Mg}+{ }_{4}^{8} \mathrm{Be}$ & 1.000 & 1.000 & 1.000 & 15.70 \\
\hline \multirow{2}{*}{$26+6$} & ${ }_{14}^{26} \mathrm{Si}+{ }_{2}^{6} \mathrm{He}$ & \multirow[t]{2}{*}{1.000} & \multirow[t]{2}{*}{1.000} & \multirow[t]{2}{*}{1.000} & 18.74 \\
\hline & ${ }_{12}^{26} \mathrm{Mg}+{ }_{4}^{6} \mathrm{Be}$ & & & & 19.07 \\
\hline $28+4$ & ${ }_{14}^{28} \mathrm{Si}+{ }_{2}^{4} \mathrm{He}$ & 1.000 & 0.169 & 0.086 & 22.40 \\
\hline $10+10$ & ${ }_{6}^{10} \mathrm{C}+{ }_{4}^{10} \mathrm{Be}$ & 1.000 & 1.000 & 1.000 & 9.94 \\
\hline $12+8$ & ${ }_{6}^{12} \mathrm{C}+{ }_{4}^{8} \mathrm{Be}$ & 1.000 & 1.000 & 1.000 & 13.68 \\
\hline \multirow{2}{*}{$14+6$} & ${ }_{8}^{14} \mathrm{O}+{ }_{2}^{6} \mathrm{He}$ & \multirow[t]{2}{*}{0.131} & \multirow[t]{2}{*}{0.290} & \multirow[t]{2}{*}{1.000} & 16.61 \\
\hline & ${ }_{6}^{14} \mathrm{C}+{ }_{4}^{6} \mathrm{Be}$ & & & & 17.12 \\
\hline $16+4$ & ${ }_{8}^{16} \mathrm{O}+{ }_{2}^{4} \mathrm{He}$ & 0.070 & 0.169 & 1.000 & 17.59 \\
\hline
\end{tabular}
$D$ gives the energetic stability of the clusterizations (in $\mathrm{MeV}$ )

The $U(3)$ symmetry of the experimentally found superdeformed, and of the theoretically predicted hyperdeformed bands of the ${ }^{36} \mathrm{Ar}$ nucleus were determined in [3]. The shell model configurations provided by the model calculations of the superdeformed [7,19], and of the hyperdeformed [8] states resulted in the $[32,12,12]$, and $[48,8,8]$ representations, respectively, while the ground state has $[20,20,12]$.

Since, the double-magic nuclei are energetically more stable, than all the others, it is reasonable to search for ternary clusterconfigurations in such a way that one of the clusters has closedshell structure. For the ${ }^{36}$ Ar nucleus two different ternary clusterizations of this kind may exist, built on the ${ }^{4} \mathrm{He}$, and ${ }^{16} \mathrm{O}$ core, respectively. We consider here only even-even clusters, again: due to energetic reasons. The results of our calculations for these configurations are summarized in Table 1. All the clusters are characterized by their ground-state $U(3)$ symmetry, i.e. they were supposed to have ground state as an intrinsic state, but collective rotations built on them were taken into account. Simple leading term approximation was used in determining their $U(3)$ symmetry (which usually coincides with the effective symmetries for light nuclei [14]).

The experimental studies on the spontaneous fission of the ${ }^{252} \mathrm{Cf}$ indicate the presence of cluster configurations in this nucleus [9]. As for the possible binary cluster-configurations, we have carried out a systematic investigation in [3]. Here we consider the ternary clusterizations of this nucleus, since however, their total number is too large due to the number of nucleons, we have to restrict ourselves to some interesting subsets. Therefore, we investigate the following cases. (i) All the possible three- 
Table 2

Ternary clusterizations of ${ }^{252} \mathrm{Cf}$ with two double magic clusters

\begin{tabular}{llllc}
\hline$C_{1}+C_{2}+C_{3}$ & \multicolumn{3}{c}{$S$} & \multirow{2}{*}{$D(1,2,3)$} \\
\cline { 2 - 4 } & $\mathrm{ND}$ & $\mathrm{SD}$ & $\mathrm{HD}$ & \\
\hline${ }^{208} \mathrm{~Pb}+{ }^{4} \mathrm{He}+{ }^{40} \mathrm{Si}$ & 0.041 & 1.000 & 0.019 & 23.32 \\
${ }^{132} \mathrm{Sn}+{ }^{78} \mathrm{Ni}+{ }^{42} \mathrm{Ca}$ & 0.010 & 0.019 & 0.039 & 19.01 \\
${ }^{132} \mathrm{Sn}+{ }^{48} \mathrm{Ca}+{ }^{72} \mathrm{Ni}$ & 0.010 & 0.019 & 0.037 & 15.87 \\
${ }^{132} \mathrm{Sn}+{ }^{16} \mathrm{O}+{ }^{104} \mathrm{Zr}$ & 0.015 & 1.000 & 1.000 & 15.16 \\
${ }^{132} \mathrm{Sn}+{ }^{4} \mathrm{He}+{ }^{116} \mathrm{Pd}$ & 0.009 & 0.064 & 1.000 & 21.33 \\
${ }^{78} \mathrm{Ni}+{ }^{78} \mathrm{Ni}+{ }^{96} \mathrm{Mo}$ & 0.009 & 0.016 & 0.027 & 17.57 \\
${ }^{78} \mathrm{Ni}+{ }^{48} \mathrm{Ca}+{ }^{126} \mathrm{Sn}$ & 0.010 & 0.018 & 0.034 & 14.87 \\
${ }^{78} \mathrm{Ni}+{ }^{40} \mathrm{Ca}+{ }^{134} \mathrm{Sn}$ & 0.010 & 0.019 & 0.037 & 20.76 \\
${ }^{78} \mathrm{Ni}+{ }^{16} \mathrm{O}+{ }^{158} \mathrm{Sm}$ & 0.026 & 1.000 & 1.000 & 11.32 \\
${ }^{78} \mathrm{Ni}+{ }^{4} \mathrm{He}+{ }^{170} \mathrm{Er}$ & 0.015 & 0.211 & 1.000 & 17.56 \\
${ }^{48} \mathrm{Ca}+{ }^{48} \mathrm{Ca}+{ }^{156} \mathrm{Ce}$ & 0.027 & 1.000 & 1.000 & 1.65 \\
${ }^{16} \mathrm{O}+{ }^{4} \mathrm{He}+{ }^{232} \mathrm{Ra}$ & 1.000 & 0.043 & 0.012 & 4.52 \\
${ }^{4} \mathrm{He}+{ }^{4} \mathrm{He}+{ }^{244} \mathrm{Pu}$ & 1.000 & 0.018 & 0.009 & 11.48 \\
\hline
\end{tabular}

cluster configurations which contain two double magic clusters. (When searching for cluster-configurations, we took into account only those nuclei, as building blocks, which have experimentally known masses [18].) (ii) Possible even-even ternary configurations, built on the ${ }^{208} \mathrm{~Pb}$ core. (iii) Ternary clusterizations, for which experimental indications exist. In doing so, we investigate not only the ground-state of ${ }^{252} \mathrm{Cf}$, but the hypothetical superdeformed and hyperdeformed states as well, which are obtained by requiring quadrupole deformation $\beta_{2}=0.6$ and 0.86 , respectively. The corresponding $U(3)$ quantum numbers are [414, 321, 303], [520, 285, 267], and [600, 260, 245] for the ground, superdeformed and hyperdeformed states, respectively [3].

The results for the ternary configurations with two doublemagic clusters are shown in Table 2. As for the possible eveneven three-cluster configurations with ${ }^{208} \mathrm{~Pb}$ core we have found the following remarkable situation. They are forbidden both in the ground state $(S \leqslant 0.06)$, and in the hyperdeformed state ( $S \leqslant 0.03$ ), but each of them is allowed in the superdeformed state. These three-cluster configurations are preferred from the energetic viewpoint $(13 \mathrm{MeV} \leqslant D(1,2,3) \leqslant 24 \mathrm{MeV})$.

Recent experimental studies, using multidetector arrays, have identified ternary clusterizations in ${ }^{252} \mathrm{Cf}$ that include ${ }^{4} \mathrm{He}$, or ${ }^{10} \mathrm{Be}$ (see for example [20] and references therein). We have performed calculations for the corresponding three-cluster configurations. The results are listed in Table 3. It is worth mentioning again, that these configurations are not allowed in the ground state from the microscopic viewpoint, some of them are allowed in the superdeformed state, and each of them is preferred in the hyperdeformed state.

To sum up: Our results show that the two aspects of the nuclear clusterization, i.e. the energetic stability and the exclusion principle have different preferences for the possible configurations. They are complementary aspects of the clusterization, and from the investigation of one of them one cannot conclude on the other. This finding is very similar to that of the similar study on binary clusterizations [3].

The present study also illustrates that the nuclear clusterization depends on the quadrupole deformation in a highly non-
Table 3

Ternary clusterizations of ${ }^{252} \mathrm{Cf}$ having some experimental indications. The $D$ values are given for configurations of even-even clusters

\begin{tabular}{llllc}
\hline$C_{1}+C_{2}+C_{3}$ & \multicolumn{3}{c}{$S$} & \multirow{2}{*}{$D(1,2,3)$} \\
\cline { 2 - 4 } & $\mathrm{ND}$ & $\mathrm{SD}$ & $\mathrm{HD}$ & \\
\hline${ }^{146} \mathrm{Ba}+{ }^{96} \mathrm{Sr}+{ }^{10} \mathrm{Be}$ & 0.014 & 1.000 & 1.000 & -0.42 \\
${ }^{130} \mathrm{Sn}+{ }^{112} \mathrm{Ru}+{ }^{10} \mathrm{Be}$ & 0.012 & 0.104 & 1.000 & 9.67 \\
${ }^{134} \mathrm{Te}+{ }^{108} \mathrm{Mo}+{ }^{10} \mathrm{Be}$ & 0.012 & 1.000 & 1.000 & 8.76 \\
\hline${ }^{156} \mathrm{Nd}+{ }^{92} \mathrm{Kr}+{ }^{4} \mathrm{He}$ & 0.013 & 1.000 & 1.000 & 8.07 \\
${ }^{150} \mathrm{Ce}+{ }^{98} \mathrm{Sr}+{ }^{4} \mathrm{He}$ & 0.013 & 1.000 & 1.000 & 5.81 \\
${ }^{148} \mathrm{Ce}+{ }^{100} \mathrm{Sr}+{ }^{4} \mathrm{He}$ & 0.012 & 1.000 & 1.000 & 6.77 \\
${ }^{148} \mathrm{Ba}+{ }^{100} \mathrm{Zr}+{ }^{4} \mathrm{He}$ & 0.012 & 1.000 & 1.000 & 5.90 \\
${ }^{146} \mathrm{Ba}+{ }^{102} \mathrm{Zr}+{ }^{4} \mathrm{He}$ & 0.011 & 0.414 & 1.000 & 6.80 \\
${ }^{144} \mathrm{Ba}+{ }^{104} \mathrm{Zr}+{ }^{4} \mathrm{He}$ & 0.011 & 0.414 & 1.000 & 8.63 \\
${ }^{141} \mathrm{Xe}+{ }^{107} \mathrm{Mo}+{ }^{4} \mathrm{He}$ & 0.011 & 0.169 & 1.000 & \\
${ }^{136} \mathrm{Te}+{ }^{112} \mathrm{Ru}+{ }^{4} \mathrm{He}$ & 0.009 & 0.069 & 1.000 & 15.20 \\
${ }^{152} \mathrm{Ce}+{ }^{96} \mathrm{Sr}+{ }^{4} \mathrm{He}$ & 0.014 & 1.000 & 1.000 & 6.42 \\
${ }^{149} \mathrm{Ce}+{ }^{99} \mathrm{Sr}+{ }^{4} \mathrm{He}$ & 0.013 & 1.000 & 1.000 & \\
${ }^{147} \mathrm{Ce}+{ }^{101} \mathrm{Sr}+{ }^{4} \mathrm{He}$ & 0.012 & 1.000 & 1.000 & \\
${ }^{147} \mathrm{Ba}+{ }^{101} \mathrm{Zr}+{ }^{4} \mathrm{He}$ & 0.011 & 1.000 & 1.000 & \\
${ }^{145} \mathrm{Ba}+{ }^{103} \mathrm{Zr}+{ }^{4} \mathrm{He}$ & 0.011 & 0.414 & 1.000 & \\
${ }^{142} \mathrm{Xe}+{ }^{106} \mathrm{Mo}+{ }^{4} \mathrm{He}$ & 0.011 & 0.290 & 1.000 & 9.26 \\
${ }^{140} \mathrm{Xe}+{ }^{108} \mathrm{Mo}+{ }^{4} \mathrm{He}$ & 0.010 & 0.120 & 1.000 & 11.00 \\
${ }^{132} \mathrm{Sn}+{ }^{116} \mathrm{Pd}+{ }^{4} \mathrm{He}$ & 0.009 & 0.075 & 1.000 & 21.33 \\
\hline${ }^{140}$ & & & &
\end{tabular}

trivial way. This conclusion is very much in line with those of other theoretical considerations [21,22]. Some aspect of this dependence can be understood based on simple geometrical arguments, like the similarity or difference in the shapes of the clusters and parent nucleus, while other aspects seem to be more hidden. Systematic investigations in order to reveal the possible role of the shell effects, for example, could be very illuminative along this line.

Another interesting phenomenon found in this work is, however, less well recognized so far. This is the appearance of the same cluster-configuration in very different states, e.g. in the ground-state and superdeformed state of a nucleus, or in the superdeformed and hyperdeformed states, or in each of these three. In the ${ }^{36} \mathrm{Ar}$ case e.g. the ${ }^{24} \mathrm{Mg}+{ }^{8} \mathrm{Be}+{ }^{4} \mathrm{He}$, ${ }^{20} \mathrm{Ne}+{ }^{12} \mathrm{C}+{ }^{4} \mathrm{He},{ }^{16} \mathrm{O}+{ }^{12} \mathrm{C}+{ }^{8} \mathrm{Be}$ clusterizations are allowed in each of the three states. Furthermore, they are energetically favored as well. (Please, note their similarity to the ${ }^{24} \mathrm{Mg}+{ }^{12} \mathrm{C}$ binary configuration, which turned out to be allowed in the ground-state, superdeformed and hyperdeformed states of ${ }^{36} \mathrm{Ar}$ as well [3]. Energetically the ternary configurations are more favored, shown by the higher values of $D(1,2,3)$ in Table 1, in comparison with the $5.135 \mathrm{MeV}$ value for the binary configuration.) The reason for this phenomenon is that if the deformation of the clusters is taken into account, and their different orientations are allowed in the description, and furthermore the effect of the antisymmetrization is included, then different spatial configurations of the same clusters may result in states of the parent nucleus with very different quadrupole configurations. This conclusion is new and unexpected in comparison with the conclusions of the phenomenological models on this phenomenon, and it turns out to be an effect which is ruled out in some approaches by some simplifying model assumptions (e.g. not taking into account triaxial deformations, 
some relative orientations, or the exclusion principle). Similar observation was made for the molecular resonances of the light nuclei when the description was elaborated enough: like in the two-center shell model [23] or in the cluster models [24,25], if unconstrained calculations were carried out.

As for the clusterization of the ${ }^{252} \mathrm{Cf}$ nucleus, indicated by the spontaneous fission experiments, we have found that most of the ternary configurations we have investigated are forbidden in the ground-state. Many of them are allowed, however, in the superdeformed and hyperdeformed states. This finding also indicates that the spontaneous fission takes place via the (tiny) component of the ground-state wave function which corresponds to an extremely deformed state, similarly to the conjecture of [26]. (Please, note that the effective quantum numbers correspond to a kind of average, which may contain contribution from many pure $S U(3)$ states, i.e. from many different quadrupole shapes.) Only the ${ }^{4} \mathrm{He}+{ }^{4} \mathrm{He}+$ core and the ${ }^{4} \mathrm{He}+{ }^{16} \mathrm{O}+$ core configurations are present in the ground state with considerable weight.

\section{Acknowledgements}

This work was supported by the OTKA (Grant Nos. T37502, T46791), and the MTA-CONACyT joint project. A.A. recognizes partial support of the János Bolyai research fellowship.

\section{References}

[1] T.M. Shneidman, G.G. Adamian, N.V. Antonenko, R.V. Jolos, W. Scheid, Phys. Rev. C 67 (2003) 014313, and references therein.

[2] B. Buck, A.C. Merchant, S.M. Perez, Phys. Rev. Lett. 76 (1996) 380, and references therein.

[3] J. Cseh, A. Algora, J. Darai, P.O. Hess, Phys. Rev. C 70 (2004) 034311.

[4] B. Buck, A.C. Merchant, S.M. Perez, Few-Body Systems 29 (2000) 53; B. Buck, A.C. Merchant, M.J. Horner, S.M. Perez, Phys. Rev. C 61 (2000) 024314.
[5] J.P. Elliot, Proc. R. Soc. A 245 (1958) 128; J.P. Elliot, Proc. R. Soc. A 245 (1958) 562.

[6] P. Rochford, D.J. Rowe, Phys. Lett. B 210 (1988) 5; D.J. Rowe, P. Rochford, J. Repka, J. Math. Phys. 29 (1988) 572.

[7] C.E. Svensson, et al., Phys. Rev. Lett. 85 (2000) 2693; C.E. Svensson, et al., Phys. Rev. C 63 (2001) 061301(R).

[8] W.D.M. Rae, A.C. Merchant, Phys. Lett. B 279 (1992) 207.

[9] J.H. Hamilton, et al., J. Phys. G 20 (1994) L85; A.V. Ramayya, et al., Phys. Rev. Lett. 81 (1998) 947.

[10] B.F. Bayman, A. Bohr, Nucl. Phys. 9 (1958/59) 596.

[11] P. Kramer, M. Moshinsky, in: E.M. Loebl (Ed.), Group Theory and Its Application, Academic Press, NY, 1968; V.G. Neudatschin, Yu. Smirnov, N.F. Golovanova, Adv. Nucl. Phys. 11 (1979) 1 ;

K.T. Hecht, Nucl. Phys. A 283 (1977) 223;

J. Draayer, Nucl. Phys. A 237 (1975) 157;

Y. Suzuki, Nucl. Phys. A 448 (1986) 395;

K. Kato, H. Tanaka, Prog. Theor. Phys. 81 (1989) 841;

J. Cseh, Phys. Rev. C 50 (1994) 2240.

[12] J. Cseh, J. Phys. G 19 (1993) L97.

[13] M. Jarrio, J.L. Wood, D.J. Rowe, Nucl. Phys. A 528 (1991) 409.

[14] P.O. Hess, A. Algora, M. Hunyadi, J. Cseh, Eur. Phys. J. A 15 (2002) 449.

[15] C. Bahri, D.J. Rowe, Nucl. Phys. A 662 (2000) 125.

[16] A. Algora, J. Cseh, J. Phys. G: Nucl. Part. Phys. 22 (1996) L39.

[17] M. Ichimura, A. Arima, E.C. Halbert, T. Terasawa, Nucl. Phys. A 204 (1973) 225;

A. Arima, in: R. Bock (Ed.), Heavy Ion Collisons, vol. 1, North-Holland, Amsterdam, 1979, p. 417.

[18] G. Audi, A.H. Wapstra, Nucl. Phys. A 595 (1995) 409.

[19] T. Bengtsson, I. Ragnarsson, Nucl. Phys. A 436 (1985) 14; A.V. Afanasjev, I. Ragnarsson, Nucl. Phys. A 591 (1995) 387.

[20] A.V. Ramayya, et al., Phys. Rev. C 57 (1998) 2370; A.V. Daniel, et al., Phys. Rev. C 69 (2004) 041305(R).

[21] G.G. Adamian, N.V. Antonenko, R.V. Jolos, Yu.V. Palchikov, W. Scheid, Phys. Rev. C 67 (2003) 054303.

[22] S. Cwiok, W. Nazarewicz, J.X. Saladin, W. Plociennik, A. Johnson, Phys. Lett. B 322 (1994) 304.

[23] H. Chandra, U. Mosel, Nucl. Phys. A 298 (1978) 151.

[24] W.D.M. Rae, Int. J. Mod. Phys. 3 (1988) 1343.

[25] J. Cseh, W. Scheid, J. Phys. G 18 (1992) 1419.

[26] G.M. Ter-Akopian, et al., Phys. Rev. Lett. 77 (1996) 32. 